Proceedings

\title{
Spectroscopic Studies Upon Silver Nanoclusters Formed on Oligonucleotides Containing a Tricyclic Cytosine Analogue, $\mathrm{tC}^{+}$
}

\author{
Agnieszka Borysowiec, Angelika Świtalska and Anna Dembska * \\ Faculty of Chemistry, Adam Mickiewicz University, Uniwersytetu Poznanskiego 8, 61-614 Poznan, Poland; \\ email1@gmail.com (A.B.); email2@gmail.com (A.Ś.) \\ * Correspondence: aniojka@amu.edu.pl \\ + Presented at the 1st International Electronic Conference on Biosensors, 2-17 November 2020; Available \\ online: https://iecb2020.sciforum.net/.
}

Published: date

\begin{abstract}
Silver nanoclusters (AgNCs) generated on DNA-templates belong to a new class of fluorescent tags showing excellent brightness, photostability as well as biocompatibility. Thus, AgNCs-DNA have been applied in various applications, from the detection of DNA/RNA, environmental monitoring to bioimaging and cancer therapy. In this work, we report fluorescent AgNCs synthesized using two 1,3-diaza-2-oxophenothiazine ( $\mathrm{tC}$ )-modified oligonucleotides that contain RET-related sequence CCCCGCCCCGCCCCGCCCCA. The communication compares the absorption and emission properties of the obtained systems with silver nanoclusters synthesized on the unmodified oligonucleotide. First, we showed the optimal conditions for AgNCs-DNA synthesis on three DNA templates: (1) RET20 with the sequence 5'-CCC CGC CCC GCC CCG CCC CA-3'; (2) RET19tC with the sequence 5'-CCC CGC CCC GCC CCG CCC tCA-3'; (3) RET14tC with the sequence 5'-CCC CGC CCC GCC CtCG CCC CA-3'. Next, the silver nanoclusters were characterized by UV/Vis absorption, fluorescence and circular dichroism spectroscopy. Silver nanoclusters RET19tC-AgNCs and RET14tC-AgNCs indicated the several times higher fluorescence intensities in the long-wave emission spectra as compared to RET20-AgNCs. Moreover, silver nanoclusters on $\mathrm{tC}$-modified oligonucleotides showed the higher stability over time. The possibility of using of the silver nanoclusters RET19tC-AgNCs for monitoring pH changes will be also tested.
\end{abstract}

Keywords: silver nanoclusters; cytosine analogue; fluorescent probe

\section{Introduction}

In recent years, an enormous progress has been made in the development of new fluorescent nanomaterials such as quantum dots (QDs) [1], nanodiamonds (NDs) [2], carbon nanodots (C-dots) [3], graphene oxide (GO) [4], carbon nanotubes (CNTs) [5], lanthanide-based upconversion nanoparticles (UCNPs) [6], luminescent metal organic framework (MOFs) [7], molecularly imprinted polymers (MIPs) [8], aggregation-induced emission dots (AIE dots) [9] or metal nanoclusters (NCs) [10].

The creation of metal nanoclusters requires an appropriate scaffold/matrix, the role of which can be played by peptides, proteins, dendrimers, polymers, thiols, and DNA fragments [10]. Due to the high binding affinity of $\mathrm{Ag}^{+}$ions to nucleobases (especially cytosines) [11,12], DNA oligonucleotides are successfully used as a template for the synthesis of silver nanoclusters [13-15]. The facile synthesis of silver nanoclusters (AgNCs) on DNA-templates as well as their excellent brightness, good photostability and biocompatibility, small size compared to other fluorophores such as quantum dots 
or organic dyes make them subject of extensive research [16,17]. Moreover, AgNCs-DNA have been applied in various applications, from the detection of metal ions, small biomolecules (for example ATP, adenosine, biological thiols, theophylline), DNA/RNA, environmental monitoring to bioimaging and cancer therapy $[10,15,16]$.

The aim of our research was to verify the influence of the fluorescent 1,3-diaza-2oxophenothiazine, $\mathrm{tC}$ on the absorption and emission properties of AgNCs synthesized by using $\mathrm{tC}-$ modified oligonucleotides that contain RET-related sequence CCCCGCCCCGCCCCGCCCCA [18]. In presented work, we compare the absorption and emission properties of the obtained systems with silver nanoclusters synthesized on the unmodified oligonucleotide with RET-related sequence. The oligonucleotides of choice are able to form four-stranded DNA structures called i-motifs [18-20]. The implications coming from fact using the folded i-motif structure as template for the synthesis of silver nanoclusters will be also presented and discussed.

\section{Materials and Methods}

The oligonucelotides used in this study were synthesized and purified by Future Synthesis (Poznan, Poland) using a high-performance liquid chromatography (Prominence HPLC) (Shimadzu, Kyoto, Japan). All other reagents were purchased from Sigma Aldrich (St. Louis, MO, USA) and were used as received. Milli-Q ultrapure water was used in all experiments.

Silver nanoclusters were synthesized following the literature procedure [14]. First, the $2.0 \mu \mathrm{M}$ solution of chosen oligonucleotide was prepared in advance in $10 \mathrm{mM}$ Tris-acetate ( $\mathrm{pH}$ 7.5. or 5.5).

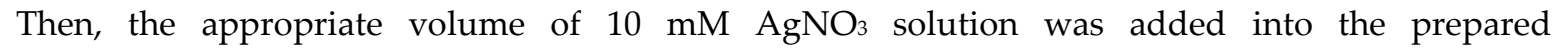
oligonucleotide solution (1:1, $\mathrm{Ag}^{+} / \mathrm{C}$ base, molar ratio or indicated as different). Next, after incubating at $4{ }^{\circ} \mathrm{C}$ for $15 \mathrm{~min}$, the freshly prepared $\mathrm{NaBH}_{4}$ solution was added to the above mixture and shaken vigorously for $1 \mathrm{~min}\left(1: 1, \mathrm{Ag}^{+} / \mathrm{NaBH}_{4}\right.$, molar ratio) to reduce silver ions and to form AgNCs on DNA. Finally, the obtained solution was further stored in the dark at $4{ }^{\circ} \mathrm{C}$ for at least $1 \mathrm{~h}$ to prepare stable DNA-AgNCs.

Spectroscopy measurements of fluorescent DNA-AgNCs were performed at $25{ }^{\circ} \mathrm{C}$ using $2 \mu \mathrm{M}$ solutions of RET-related oligonucleotides in $10 \mathrm{mM}$ Tris-acetate buffer (pH 7.5 or 5.5) as follows:

Absorption spectra of samples were recorded in the spectral range of 200-800 $\mathrm{nm}$ by means of Jasco V-750 spectrophotometer (Jasco, Tokyo, Japan).

CD spectra of samples were recorded on a Jasco J-820 Spectropolarimeter (Jasco, Tokyo, Japan) with connected a temperature controller (PTC-423L). The CD spectra were obtained by taking the average of three scans in the range of $400-200 \mathrm{~nm}$, with a scan rate of $500 \mathrm{~nm} / \mathrm{min}$.

Fluorescence spectra were measured on a Jasco spectrofluorimeter FP-8200 (Jasco, Tokyo, Japan) with $10 \mathrm{~nm}$ excitation and $10 \mathrm{~nm}$ emission slits and were carried out using $0.4 \times 1 \mathrm{~cm}$ quartz cuvettes containing $1 \mathrm{ml}$ of solution. Emission and excitation spectra of samples were recorded in the 270-750 $\mathrm{nm}$ range using $\lambda \mathrm{ex}=260 \mathrm{~nm}, \lambda \mathrm{ex}=395 \mathrm{~nm}, \lambda \mathrm{ex}=550 \mathrm{~nm}$ and $\lambda \mathrm{em}=605 \mathrm{~nm}$, respectively.

\section{Results}

The DNA template used for synthesis of silver nanoclusters (AgNCs) is cytosine-rich (C-rich) oligonucleotide related to the RET sequence: 5'-CCCCGCCCCGCCCCGCCCCA-3'(RET20) [18]. In this work, we also report fluorescent AgNCs synthesized on tC-modified oligonucleotides that contain the same RET-related sequence: 5'-CCCCGCCCCGCCCCGCCCtCA-3' (RET19tC) and 5'CCCCGCCCCGCCCtCGCCCCA-3' (RET14tC) [20]. 1,3-diaz-2-oxophenoxazine, tC is a tricyclic cytosine analog, which is a highly fluorescent marker with an absorption maximum at $385 \mathrm{~nm}$ and an emission maximum at $500 \mathrm{~nm}$ [21]. Each C-rich oligonucleotide of choice in this study is able to form four-stranded structure (an i-motif) in acidic solutions. In our previous work, we indicated that $\mathrm{pH}$ value of conformational transition ( $\mathrm{pH}$ ) of RET20 (unmodified) is equal of 7.1 [20]. Therefore, we performed optimization of RET20 silver nanoclusters (RET20-AgNCs) syntesis in buffer solutions, pH 7.5. 


\subsection{Optimization Conditions of RET20-AgNCs Synthesis}

The first synthesis of AgNCs on a DNA template rich in cytosine bases (5'-AGGTCGCCGCCC$\left.3^{\prime}\right)$ was performed by Dickson et al. in 2004 [13]. Since then it has been proven that DNA-AgNCs emission can be controlled by changing the experimental conditions (i.e. $\mathrm{pH}$, type of buffer, salt addition, concentration of reagents) $[22,23]$ or using DNA sequences that differ in length, composition and structure [24,25]. We synthesized the RET20-AgNCs nanoclusters by adding sodium borohydride to the mixture of silver nitrate and the oligonucleotide with RET-related sequence CCCCGCCCCGCCCCGCCCCA under air, as described in the literature [14]. The formation of the nanoclusters during the reduction step was evident from the appearance of a yellowish color. Several electronic transitions are also observed in the absorption spectra of the RET20-AgNCs nanoclusters (Figure 1a). A primary distinguishing feature of the nanoclusters is their strong peak at $440 \mathrm{~nm}$ that is red-shifted and narrower relative to the plasmon transition of the nanoparticles, which only displays one broad peak with amaximum at $405 \mathrm{~nm}$ [14]. Figure $1 \mathrm{~b}$ shows the emission spectra of the as-prepared RET20-AgNCs with excitation at $260 \mathrm{~nm}$, which is near the absorption maxima of DNA bases. Initially, the spectrum is dominated by the red emission with an apparent maximum at $\lambda$ max ca. $600 \mathrm{~nm}$ and a shoulder at lower wavelengths (black line, Figure 1b). Moreover, the evaluation of the emission spectra over time indiates that "red emission" is decreasing in contrary to "green emission". Two bands with different spectral parameters ("green" and "red" emitters) have been previously observed and assigned to small silver nanoclusters according to theoretical and experimental studies [14,26-28].

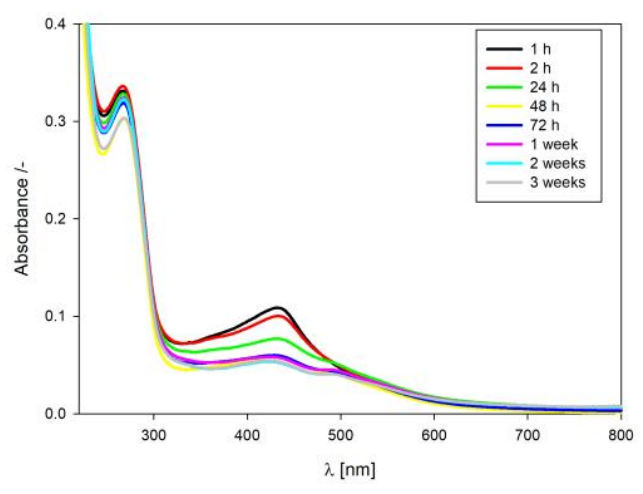

(a)

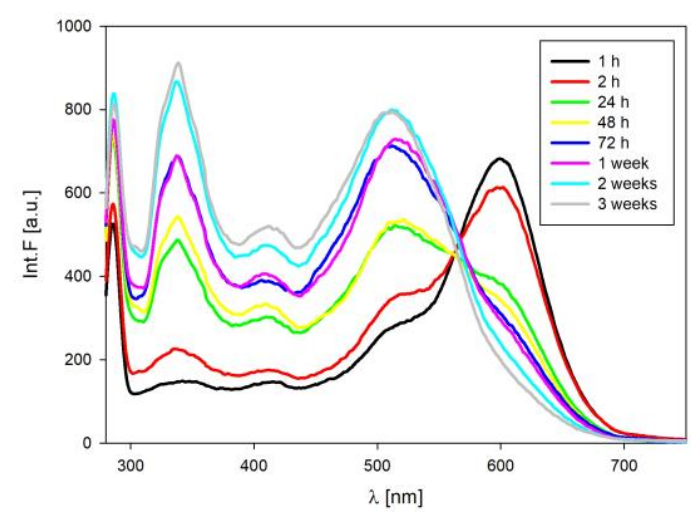

(c)

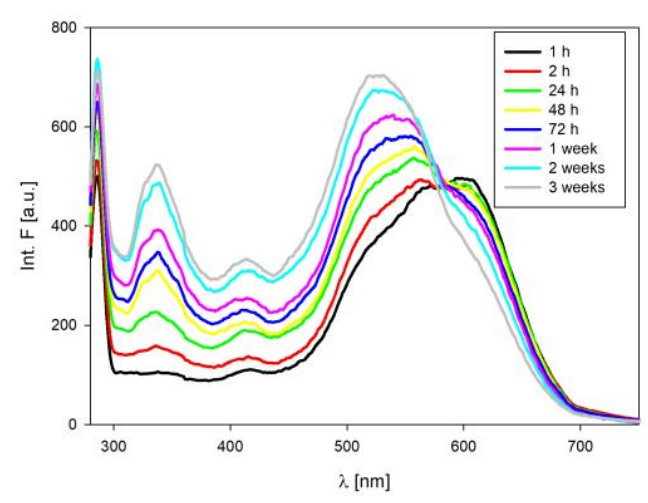

(b)

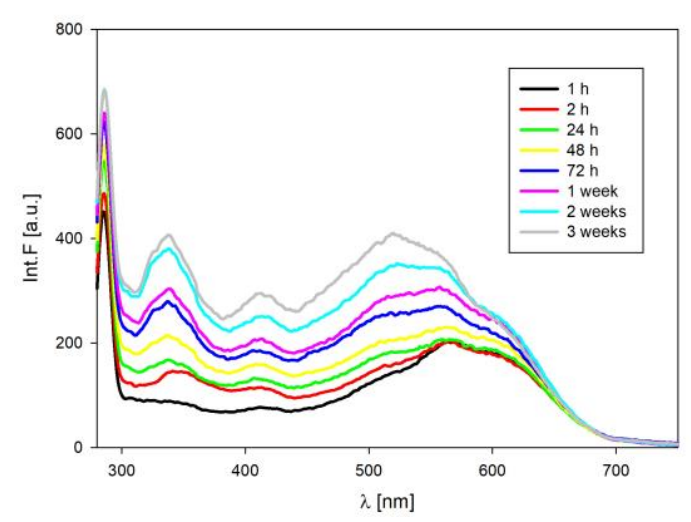

(d)

Figure 1. Spectroscopic spectra of RET20-AgNCs prepared using different $\mathrm{Ag}^{+}$to cytosine ratio: (a) absorption spectra, 1:1 $\mathrm{Ag}^{+} / \mathrm{C}$; (b) emission spectra, 1:1 $\mathrm{Ag}^{+} / C$; (c) emission spectra, 0.5:1 $\mathrm{Ag}^{+} / \mathrm{C}_{\text {; }}$ (d) 
emission spectra, 2:1 Ag+C. Excitation wavelength set at $\lambda \mathrm{ex}=260 \mathrm{~nm}$. The samples were prepared in Tris-acetate buffer solution (10 mM, pH 7.5).

Knowing that the $\mathrm{Ag}^{+} / \mathrm{DNA}$ molar ratio determines the fluorescence intensity [29-31], we tested the three different $\mathrm{Ag}^{+} / \mathrm{DNA}$ molar ratios for preparation of the RET20-AgNCs nanoclusters. The RET20-AgNCs were prepared at 1:1, 0.5:1, 2:1 molar ratios of $\mathrm{Ag}^{+} /$cytosine (C) bases. As expected, the $\mathrm{Ag}^{+} / \mathrm{C}$ molar ratio influenced on RET20-AgNCs fluorescence intensity as well as proportion between "red" and "green" emission (compare black lines in Figure 1b-d). Moreover, the emission spectra of RET20-AgNCs with excitation at $550 \mathrm{~nm}$ revealed that nanoclusters prepared at a 1:1 $\mathrm{Ag}^{+} / \mathrm{C} \mathrm{molar}$ ratio showed the highest fluorescence intensity values after 24 since reduction comparing with ones prepared at $0.5: 1$ or 2:1 molar ratio $\left(\mathrm{Ag}^{+} / \mathrm{C}\right)$.

As mentioned, depending on the preparation conditions, the AgNCs on a DNA template can exhibit different photoluminescence characteristics. Therefore, we not only tested the effect of the $\mathrm{Ag}^{+} / \mathrm{DNA}$ molar ratio on spectral properties of RET20-AgNCs, but also the effects of:

- $\quad \mathrm{Ag}^{+}$to sodium nitrate molar ratio

- $\quad$ time gap between addition of $\mathrm{Ag}^{+}$and sodium nitrate to RET20 in buffer solution

- $\quad$ RET20 concentration

As results, the following conditions were selected: DNA/Ag+ 1:1 molar ratio; $2 \mu \mathrm{M}$ DNA; $\mathrm{Ag}^{+} / \mathrm{BH}_{4}^{-1}$ 1:1 molar ratio, 15 minutes gap time between addition of $\mathrm{AgNO}_{3}$ and $\mathrm{NaBH}_{4}$ to $\mathrm{DNA}$ template solution. After selecting the optimal synthesis conditions, we conducted the experiments to assess the effect of $\mathrm{tC}$ (1,3-diaza-2-oxophenothiazine) substitution position (modified sequences) on formation and emission of RET20-AgNCs.

\subsection{Effect of tC on RET20-AgNCs Nanoclusters}

For our purpose, we used two tC-modified fluorescent oligonucleotides contain RET-related sequence CCCCGCCCCGCCCCGCCCCA. The oligonucleotides are named RET14tC and RET19tC due to the position of $\mathrm{tC}$ analogue in the chain.

\subsubsection{Monitoring the Formation of Nanoclusters on RET-Related Templates}

We measured circular dichroism (CD) and UV-Vis absorption spectra to proof the formation of silver nanoparticles templated by tC- modified RET20 oligonucleotides. CD spectra provide diagnostic signatures for the presence of secondary structures in the single stranded DNA sequence [32]. A characteristic positive peak at $\sim 288 \mathrm{~nm}$ and a negative peak at $\sim 263 \mathrm{~nm}$, with crossover at $\sim 273$ $\mathrm{nm}$, indicate the formation of an i-motif structure (Figure 2a) [32-34]. As seen in Figure 2c,d, the absorption spectra of freshly prepared AgRET19tC-AgNCs and RET14tC-AgNCs exhibit the peak with local maximum at $440 \mathrm{~nm}$ and $425 \mathrm{~nm}$, respectively. In both cases, long-wave absorption band at $\sim 500 \mathrm{~nm}$ is observed within $24 \mathrm{~h}$ after reduction step. These bands are all considered indicative of genuine nanoclusters because they are not present in case of silver nanoparticles formation, which only absorb with one broad peak at $\lambda \max =405 \mathrm{~nm}$ [14]. Moreover, in the case of RET19tC-AgNCs, the intensity of the band at a wavelength of about $400 \mathrm{~nm}$ have increased within time and after another $24 \mathrm{~h}$ is even higher than the band at $425 \mathrm{~nm}$. nanoclusters. Analogous to the evolution of the absorption spectra, the CD spectra also change upon reduction of the $\mathrm{Ag}^{+}$(Figure 2). The differences between the spectra in Figure 2 indicate that the silver nanoclusters induce different structural changes in RET20, RET14tC and RET19 tC samples than does Ag+. First, the CD spectra of RET20, RET14tC and RET19 tC in buffer solution pH 7.5 (black line, Figure 2b,d,f) show the positive peak around $280 \mathrm{~nm}$ coming from predominantly unstructured cytosine oligonucleotides [33,35]. Binding of $\mathrm{Ag}^{+}$with the cytosines significant affects the DNA conformation, that manifests as two strong peaks with negative ellipticity develop at $220 \mathrm{~nm}$ and $265 \mathrm{~nm}$ and positive peak shifted to $290 \mathrm{~nm}$ (red line, Figure $2 b, d, f)$. These results are consistent with our previous studies upon silver nanoclusters templated on oligonucleotides containing twelve cytosines [36]. The reduction of $\mathrm{Ag}^{+}$ ions by $\mathrm{BH}_{4}^{-1}$ only gives a decrease in the intensity of negative bands (220 nm and $265 \mathrm{~nm}$ ) (green 
line, Figure $2 b, d, f)$. These elliptical changes suggest that nanoclusters retain the chirality of the DNA template.

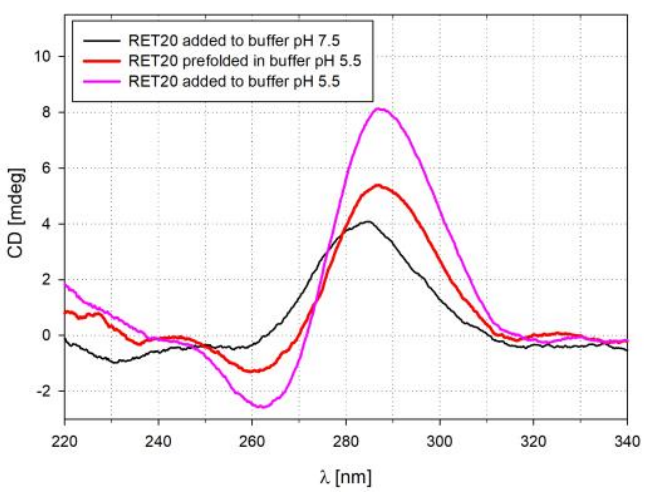

(a)

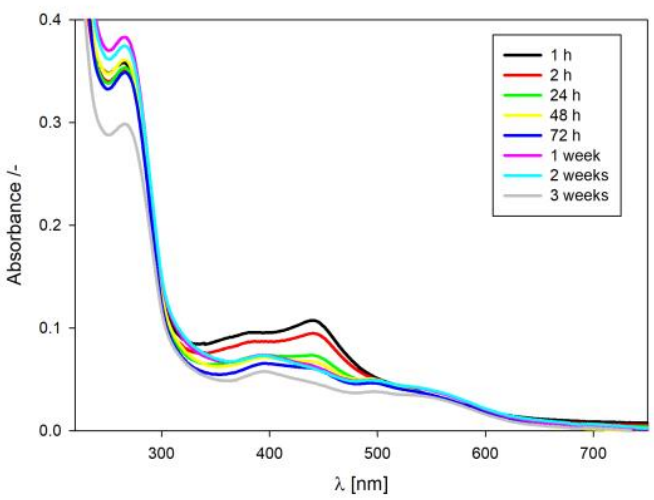

(c)

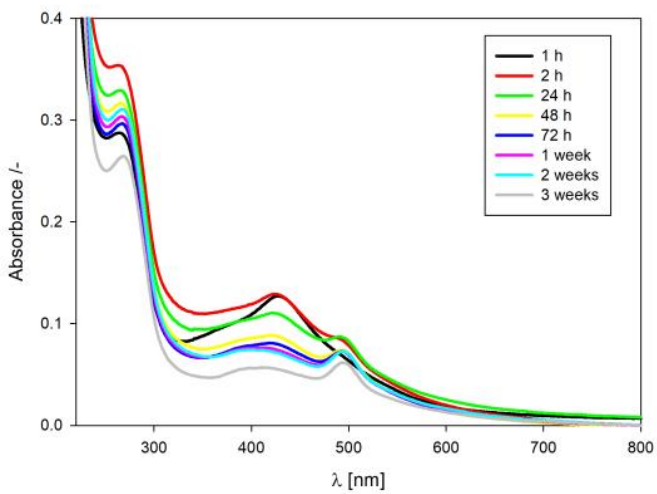

(e)

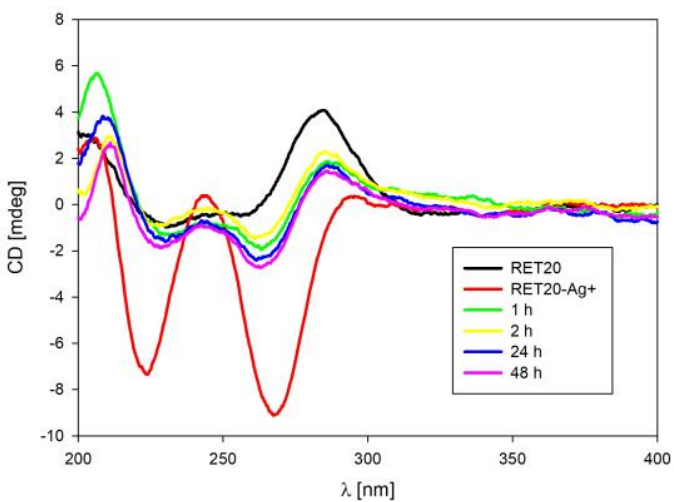

(b)

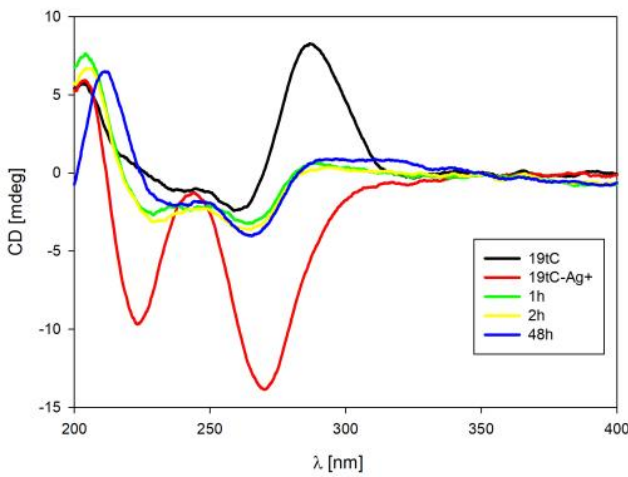

(d)

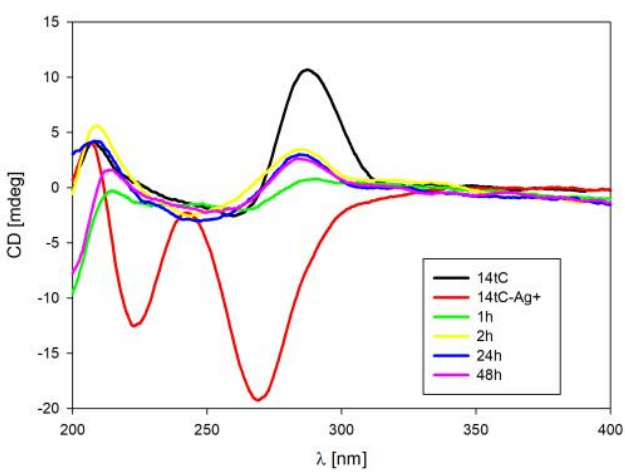

(f)

Figure 2. Monitoring the structural changes during formation of AgNCs: (a) CD spectra of RET20 in acidic and alkaline buffers; (b) CD spectra of RET20-AgNCs (c) absorption spectra of RET19tCAgNCs; (d) CD spectra of RET19tC-AgNCs (e) absorption spectra of RET14tC-AgNCs (f) CD spectra of RET14tC-AgNCs. All AgNCs were prepared in Tris-acetate buffer solution (10 mM, pH 7.5) using $1: 1 \mathrm{Ag}^{+} / \mathrm{C}$. 


\subsubsection{Fluorescence Properties of Nanoclusters on RET-Related Templates}

The excitation of tC-modified RET20 in slightly basic solution by using $260 \mathrm{~nm}$ results fluorescence with two strong emission bands with $\lambda \max =348 \mathrm{~nm}$ and $\lambda \max =509 \mathrm{~nm}$ (Figure 3a,c). It is also worth noting that the fluorescence intensities of RET19tC-AgNCs as well as RET14tC-AgNCs are several times higher than RET20-AgNCs (compare Figure 3a or 3c with Figure 1b). Except of the major peak situated at $\lambda \max =509 \mathrm{~nm}$ in emission spectra of RET19tC-AgNCs (Figure 3a), one can notice a shoulder at higher wavelengths that confirms the presence of "red" fluorescence as well. The small amount of "red" fluorescence was also detected in the emission spectra of RET14tC-AgNCs excited directly at $550 \mathrm{~nm}$ (data not shown). All tested AgNCs were also excited at $\lambda$ ex $=395 \mathrm{~nm}$, as this wavelength is absorbed by $\mathrm{tC}$. The obtained emission spectra indicated that the strong fluorescence of RET19tC-AgNCs as well as of RET14tC-AgNCs coming from excited tC is affected by addition of $\mathrm{Ag}^{+}$(red line, Figure $3 \mathrm{~b}, \mathrm{~d}$ ). In both cases, the binding of $\mathrm{Ag}^{+}$causes slightly red-shift and decreasing of $\mathrm{tC}$ emission spectra. These results are in good agreement with previous results indicating that protonation of $\mathrm{tC}$ fluorophore $\left(\mathrm{tC}-\mathrm{H}^{+}\right)$is accompanied with red-shift as well as decreasing in fluorescence intensity. Further reduction of $\mathrm{Ag}^{+}$to $\mathrm{Ag}^{0}$ and nanoclusters formation is accompanied with quenching fluorescence emission of $\mathrm{tC}$ (green line, Figure $3 \mathrm{~b}, \mathrm{~d}$ ). Comparing the emission spectra of RET14tC-AgNCs (exciting at $395 \mathrm{~nm}$ or $260 \mathrm{~nm}$ ) with RET20-AgNCs it seems that in case of RET14tC-AgNCs the "green" fluorescence comes rather from $\mathrm{Ag} / \mathrm{tC}$ than from $\mathrm{Ag} /$ cytosines nanoclusters. The same hypothesis can be assigned to RET19tC-AgNCs.

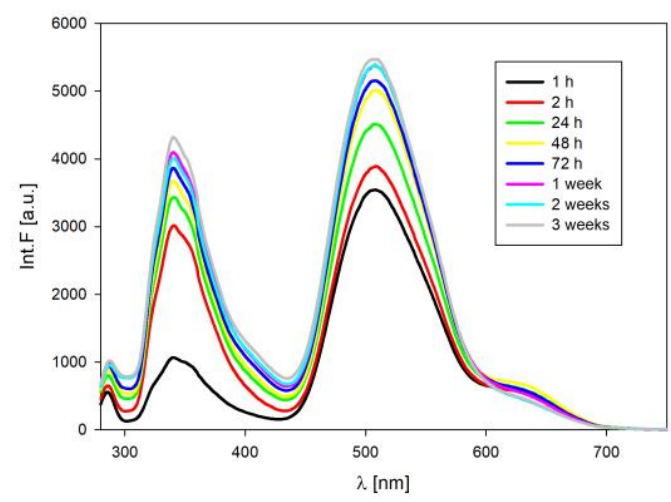

(a)

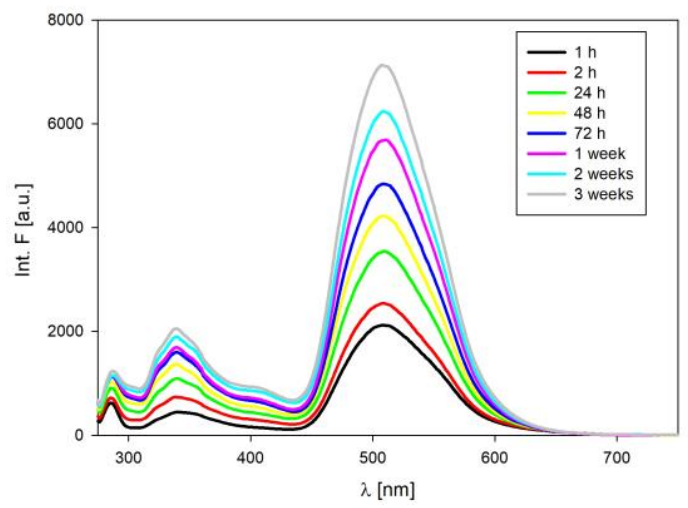

(c)

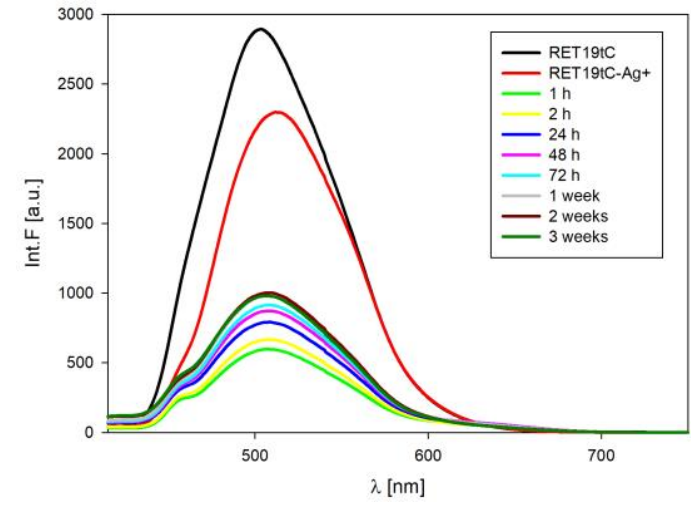

(b)

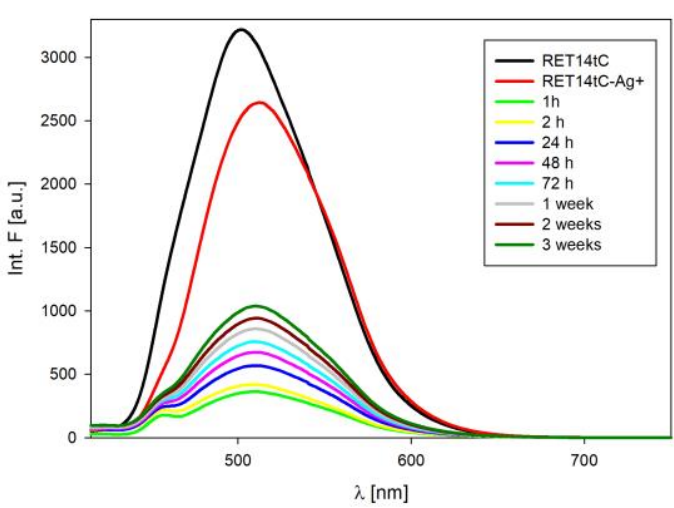

(d)

Figure 3. Fluorescence spectra of AgNCs obtained on tC-modified RET20 oligonucleotides: (a) emission spectra of RET19tC-AgNCs, excited at $260 \mathrm{~nm}$; (b) emission spectra of RET19tC-AgNCs, excited at $395 \mathrm{~nm}$; (c) emision spectra of RET14tC-AgNCs, excited at $260 \mathrm{~nm}$; (d) emission spectra of 
RET14tC-AgNCs, excited at 395nm. The samples were prepared in Tris-acetate buffer solution (10 $\mathrm{mM}, \mathrm{pH}$ 7.5).

\subsection{I-Motif as Template for Silver Nanoclusters}

The synthesis of fluorescent silver nanoclusters on RET20 as well as RET19tC template was proceeded by the i-motifs formation in acidic conditions (buffer solution pH 5.5). As previously, the AgNCs formation was manifested by the observable colour change of the solution from transparent into yellowish one within just a few hours after the addition of $\mathrm{AgNO}_{3}$ and $\mathrm{NaBH}_{4}$ as reducing agent to the folded i-motifs. I-motif-templated silver nanoclusters fluoresce as several emission bands clearly seen upon excitation at $260 \mathrm{~nm}$ wavelength. Therefore, a few emission bands with $\lambda$ max $c a$. = $338 \mathrm{~nm}, \lambda \max c a .415 \mathrm{~nm}, \lambda \max c a .525 \mathrm{~nm}$ with shoulder at $c a .605 \mathrm{~nm}$ were obtained for freshly prepared RET20-AgNCs (Figure 4a); whereas for RET19tC-AgNCs only two emission bands were seen with $\lambda \max c a .348 \mathrm{~nm}$ and $\lambda \max c a .509 \mathrm{~nm}$ (Figure 4c). Importantly, the overall fluorescence of RET19tC-AgNCs is several times higher than that of RET20-AgNCs regardless of the initial secondary structure used (please compare Figures 1a with 3a or Figure 4a with 4c). Both RET20-AgNCs and RET19tC-AgNCs nanoclusters (in buffer $\mathrm{pH}$ 5.5) were excited at $\lambda \mathrm{ex}=395 \mathrm{~nm}$, which is the wavelength absorbed by the fluorescent cytosine derivative $t C$ (Figure 4b,d). As expected, tCmodified RET20 in acidic solution shows emission band with $\lambda$ max $=520 \mathrm{~nm}$ coming from tC- $\mathrm{H}^{+}$ fluorophore (black line, Figure 4d) [20]. Note that addition of $\mathrm{Ag}^{+}$causes slightly blue-shift and remarkable enhancement (four times higher intensity) of tC emission spectrum (red line, Figure $4 \mathrm{~d}$ ). These results also indicate that $\mathrm{Ag}^{+}$replaces $\mathrm{H}^{+}$bound to $\mathrm{tC}$ fluorophore, that is in good agreement with previous results indicating that protonation of $\mathrm{tC}$ fluorophore $\left(\mathrm{tC}-\mathrm{H}^{+}\right)$is accompanied with redshift and decreasing in fluorescence intensity [20]. Further reduction of $\mathrm{Ag}^{+}$to $\mathrm{Ag}^{0}$ and RET19tCAgNCs nanoclusters formation is manifested by quenching fluorescence emission almost to the initial level, however without red-shift. Comparing the emission spectra of RET19tC-AgNCs (excited at 395 $\mathrm{nm}$ or $260 \mathrm{~nm}$ ) with RET20-AgNCs it seems that in case of RET19tC-AgNCs only slightly amount of "green" fluorescence comes from $\mathrm{Ag}^{0}$ /cytosines nanoclusters.

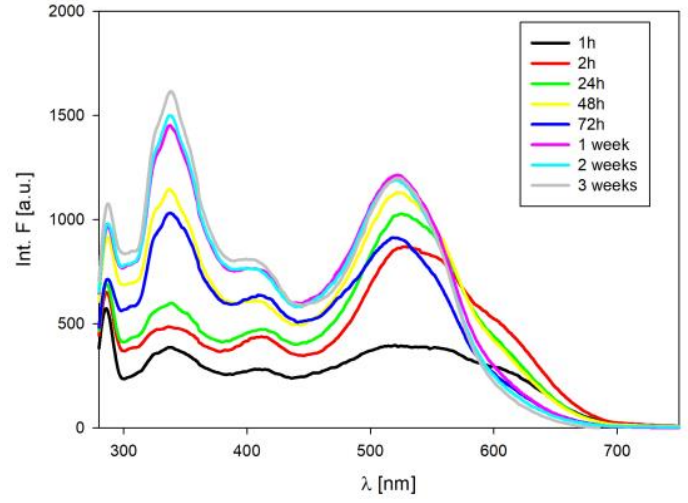

(a)

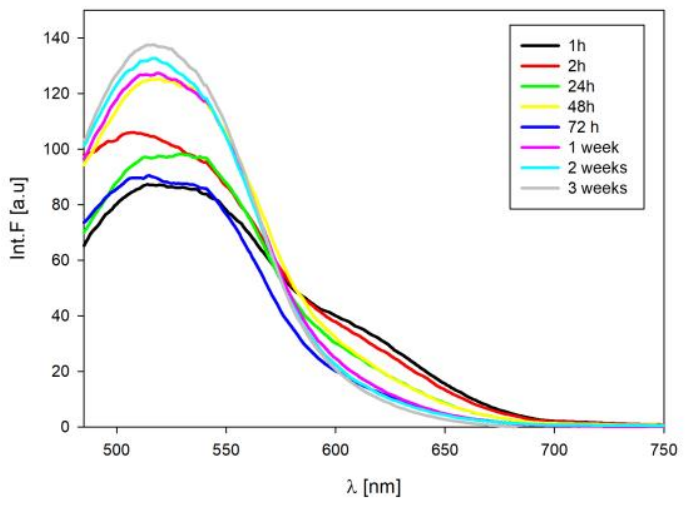

(b) 


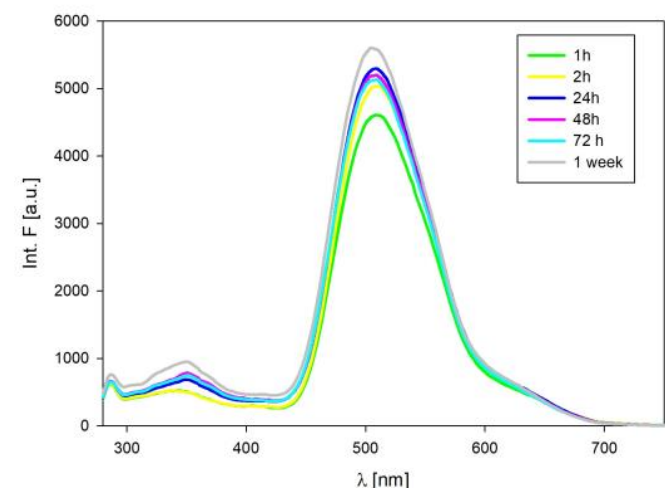

(c)

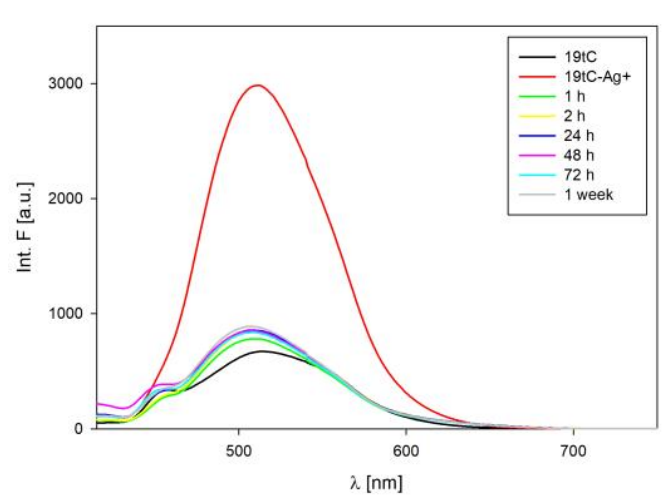

(d)

Figure 4. Fluorescence spectra of AgNCs obtained on i-motifs: (a) emission spectra of RET20-AgNCs, excited at $260 \mathrm{~nm}$; (b) emission spectra of RET20-AgNCs, excited at $395 \mathrm{~nm}$; (c) emission spectra of RET19tC-AgNCs, excited at $260 \mathrm{~nm}$; (d) emission spectra of RET14tC-AgNCs, excited at $395 \mathrm{~nm}$. The i-motifs were folded a day before in Tris-acetate buffer solution (10 mM, pH 5.5). All AgNCs were prepared using 1:1 $\mathrm{Ag}^{+} / \mathrm{C}$.

\section{Discussion}

DNA-AgNCs silver nanoclusters were synthesized on three single stranded DNA (ssDNA) templates with the RET-related sequence: $\mathrm{C}_{4} \mathrm{GC}_{4} \mathrm{GC}_{4} \mathrm{GC}_{4} \mathrm{~A}$, two of which contained the single cytosine fluorescent analogue 1,3-diaza-2-oxophenothiazine $(\mathrm{tC})$ substituted at 14th or 19th position from the $5^{\prime}$ end. The presence of $\mathrm{tC}$ in the oligonucleotide sequence has a significant influence not only on the spectroscopic properties of RET-AgNCs, but also on their stability. After the addition of $\mathrm{NaBH}_{4}$ to the $\mathrm{Ag}^{+}-\mathrm{RET}$ complex, the high reduction capacity of the solution promotes the formation of reduced silver nanoclusters with "green" as well as "red" emission. The fluorescence intensities of RET20-AgNCs prepared at slightly alkaline $\mathrm{pH}$ are higher than ones obtained in an acidic environment and regardless of the buffer solution $\mathrm{pH}$, population of "red" emitting RET20-AgNCs $(605 \mathrm{~nm})$ decreases contrary to increasing population of "green" emitting RET20-AgNCs $(520 \mathrm{~nm})$. The emission spectra of RET19tC-AgNCs and RET14tC-AgNCs exhibited fluorescence several times higher in the long-term range than RET20-AgNCs. The reduction of $\mathrm{Ag}^{+}$to $\mathrm{Ag}^{0}$ in RET14tC- $\mathrm{Ag}^{+}$or RET19tC - Ag+ complexes leads to the nanoclusters formation, which is manifested by fluorescence quenching. Comparing the emission spectra of RET14tC-AgNCs or RET19tC-AgNCs (excited at 395 $\mathrm{nm}$ or $260 \mathrm{~nm}$ ) with RET20-AgNCs, it seems that in case of AgNCs templated on tC-modified ssDNA only slightly amount of "green" fluorescence comes from $\mathrm{Ag}$ /cytosines nanoclusters.

Taking into account our previous studies revealing that analytical response of probes based on RET14tC as well as RET19tC oligonucleotides to $\mathrm{pH}$ changes depends on the difference between fluorescence quantum yield of tC in ssDNA and in i-motif [20], we decided to test ability of silver nanoclusters templated on mentioned oligonucleotides to serve as $\mathrm{pH}$ probe. Therefore, AgNCs obtained on RET19tC template, prefolded in acidic buffer solution were titrated with $0.5 \mathrm{M} \mathrm{NaOH}$. The preliminary results indicated that system is not sensitive to small $\mathrm{pH}$ changes, however increase in fluorescence signal was observed in clearly higher $\mathrm{pH}$ value. In order to learn more about the synthesized AgNCs and to evaluate their potential as sensor, additional experiments should be performed, such as the measurement of subsequent absorption, fluorescence and CD spectra of RET19tC-AgNCs and RET14tC-AgNCs in different temperatures and $\mathrm{pH}$ solutions (dynamic approach).

What is important, we observed that the presence of tC in RET oligonucleotide results in the highest stability of RET19tC-AgNCs. In view of these advantages, it is worth introducing the tC tag into the oligonucleotide sequence as it will not disturb the original structure. This means that the 
stability of DNA-AgNCs can be preserved and even better regardless of the fact that cytosine has been replaced by $\mathrm{tC}$.

\section{Conclusions}

Further studies concerning the influence the position of the fluorescent cytosine analogue (for example, $\mathrm{tC}$ ) in cytosine-rich ssDNA template (especially folded into i-motif) on stability as well as distribution of "green" and "red" emitting population of fluorescent silver nanoclusters should be undertaken.

Author Contributions: A.D. conceived and designed the study. A.D. and A.S. supervised the project. A.B. carried out experiments and prepared first version of figures. All authors analyzed the data. A.D. interpreted data and wrote the manuscript. All authors approved the final version of the manuscript. All authors have read and agreed to the published version of the manuscript.

Acknowledgments: Authors thank Patrycja Bielecka for providing oligonucleotides used in this study.

Conflicts of Interest: The authors declare no conflict of interest.

\section{References}

1. Petryayeva, E.; Algar, W.R.; Medintz, I.L. Quantum Dots in Bioanalysis: A Review of Applications Across Various Platforms for Fluorescence Spectroscopy and Imaging. Appl. Spectrosc. 2013, 67, 215-252.

2. Mochalin, V.N.; Shenderova, O.; Ho, D.; Gogotsi, Y. The Properties and Applications of Nanodiamonds. Nat. Nanotechnol. 2012, 7, 11-23.

3. Baker, S.N.; Baker, G.A. Luminescent Carbon Nanodots: Emergent Nanolights. Angew. Chem. Int. Ed. 2010, $49,6726-6744$.

4. Loh, K.P.; Bao, Q.L.; Eda, G.; Chhowalla, M. Graphene Oxide as a Chemically Tunable Platform for Optical Applications. Nat. Chem. 2010, 2, 1015-1024.

5. Wu, H.C.; Chang, X.L.; Liu, L.; Zhao, F.; Zhao, Y.L. Chemistry of Carbon Nanotubes in Biomedical Applications. J. Mater. Chem. 2010, 20, 1036-1052.

6. Wang, F.; Banerjee, D.; Liu, Y.S.; Chen, X.Y.; Liu, X.G. Upconversion Nanoparticles in Biological Labeling, Imaging, and Therapy. Analyst 2010, 135, 1839-1854.

7. Zhu, S.-Y.; Yan, B. A novel sensitive fluorescent probe of $\mathrm{S}_{2} \mathrm{O}_{8}{ }^{2}$ and $\mathrm{Fe}^{3+}$ based on covalent postfunctionalization of a zirconium(iv) metal-organic framework. Dalton Trans. 2018, 47, 11586-11592.

8. Wang, J.; Wang, Y.; Qiu, H.; Sun, L.; Dai, X.; Pan, J.; Yan, Y. A Novel Sensitive Luminescence Probe Microspheres for Rapid and Efficient Detection of $\tau$-Fluvalinate in Taihu Lake. Sci. Rep. 2017, 7, 46635.

9. Li, H.; Wang, C.; Hou, T.; Li, F. Amphiphile-Mediated Ultrasmall Aggregation Induced Emission Dots for Ultrasensitive Fluorescence Biosensing. Anal. Chem. 2017, 89, 9100-9107.

10. Zhang, Q.; Yang, M.; Zhu, Y.; Mao C. Metallic Nanoclusters for Cancer Imaging and Therapy. Curr. Med. Chem. 2018, 25, 1379-1396.

11. Soto-Verdugo, V.; Metiu, H.; Gwinn, E. The Properties of Small Ag Clusters Bound to DNA Bases. J. Chem. Phys. 2010, 132, 195102.

12. Ono, A.; Cao, S.; Togashi, H.; Tashiro, M.; Fujimoto, T.; Machinami, T.; Oda, S.; Miyake, Y.; Okamoto, I.; Tanaka Y. Specific Interactions between Silver(i) Ions and Cytosine-Cytosine Pairs in DNA Duplexes. Chem. Commun. 2008, 39, 4825-4827.

13. Petty, J.T.; Zheng, J.; Hud, N.V.; Dickson, R.M. DNA Templated AgAg Nanocluster Formation. J. Am. Chem. Soc. 2004, 126, 5207-5212.

14. Ritchie, C.M.; Johnsen, K.R.; Kiser, J.R.; Antoku, Y.; Dickson, R.M.; Petty. J.T. Ag Nanocluster Formation Using a Cytosine Oligonucleotide Template. J. Phys. Chem. C 2007, 111, 175-181.

15. Yuan, Z.; Chen, Y.-C.; Li, H.-W.; Chang, H.-T. Fluorescent silver nanoclusters stabilized by DNA scaffolds, Chem. Commun. 2014, 50, 9800-9815.

16. Chen, Y.; Phipps, L.M.; Werner, J.H.; Chakraborty, S.; Martinez, J.S. DNA Templated Metal Nanoclusters: From Emergent Properties to Unique Applications. Acc. Chem. Res. 2008, 51, 12756-12763.

17. New, S.Y.; Lee, S.T.; Su, X.D. DNA-templated silver nanoclusters: Structural correlation and fluorescence modulation. Nanoscale 2016, 8, 17729-17746. 
18. Guo, K.; Pourpak, A.; Beetz-Rogers, K.; Gokhale, V.; Sun, D.; Hurley, L.H. Formation of Pseudosymmetrical G-Quadruplex and i-Motif Structures in the Proximal Promoter Region of the RET Oncogene. J. Am. Chem. Soc. 2007, 129, 10220-10228.

19. Dembska, A.; Bielecka, P.; Juksowiak, B. pH-Sensing fluorescence oligonucleotide probes based on an imotif scaffold: A review. Anal. Methods 2017, 9, 6092-6106.

20. Bielecka, P.; Dembska, A.; Juskowiak, B. Monitoring of pH Using an i-Motif-Forming Sequence Containing a Fluorescent Cytosine Analogue, tC. Molecules 2019, 24, 952.

21. Preus, S.; Kilså, K.; Wilhelmsson, L.M.; Albinsson, B. Photophysical and structural properties of the fluorescent nucleobase analogues of the tricyclic cytosine (tC) family. Phys. Chem. Chem. Phys. 2010, 12, 8881-8892.

22. Deng, H.; Yu, H.T. A Mini Review on Controlling the Size of Ag Nanoclusters by Changing the Stabilizer to Ag Ratio and by Changing DNA Sequence. Adv. Nat. Sci. 2015, 8, 1-9.

23. Xie, Y.-P.; Shen, Y.-L.; Duan, G.-X.; Han, J.; Zhang, L.-P.; Lu X. Silver nanoclusters: Synthesis, structures and photoluminescence. Mater. Chem. Front. 2020, 4, 2205-2222S.

24. Copp, M.; Schultz, D.E.; Swasey, S.; Gwinn, E.G. Atomically precise arrays of fluorescent silver clusters: A modular approach for metal cluster photonics on DNA nanostructures. ACS Nano 2015, 9, 2303-2310.

25. O'Neill, P.R.; Young, K.; Schiffels, D.; Fygenson, D.K. Few-atom fluorescent silver clusters assemble at programmed sites on DNA nanotubes. Nano Lett. 2012, 12, 5464-5469.

26. Rabin, I.; Schulze, W.; Ertl, G. Light emission during the agglomeration of silver clusters in noble gas matrixes. J. Chem. Phys. 1998, 108, 5137-5142.

27. Fedrigo, S.; Harbich, W.; Buttet, J. Optical response of Ag2, Ag3, Au2, and Au3 in argon matrixes. J. Chem. Phys. 1993, 99, 5712-5717.

28. Bonacic-Koutecky, V.; Pittner, J.; Boiron, M.; Fantucci, P. An accurate relativistic effective core potential for excited states of Ag atom: An application for studying the absorption spectra of Agn and Agn(+) clusters. J. Chem. Phys. 1999, 110, 3876-3886.

29. Lan, G.-Y.; Huang, C.-C.; Chang, H.-T. Silver nanoclusters as fluorescent probes for selective and sensitive detection of copper ions. Chem. Commun. 2010, 46, 1257-1259.

30. Richards, C.I.; Choi, S.; Hsiang, J.C.; Antoku, Y.; Vosch, T.; Bongiorno, A.; Tzeng, Y.L.; Dickson, R.M. Oligonucleotide stabilized Ag nanocluster fluorophore. J. Am. Chem. Soc. 2008, 130, 5038-5039.

31. Deng, H.; Yu, H.T. A Mini Review on Controlling the Size of Ag Nanoclusters by Changing the Stabilizer to Ag Ratio and by Changing DNA Sequence. Adv. Nat. Sci. 2015, 8, 1-9.

32. Kypr, J.; Kejnovská, I.; Renciuk, D.; Vorlícková, M. Circular dichroism and conformational polymorphism of DNA. Nucleic Acids Res. 2009, 37, 1713-1725.

33. Simonsson, T.; Pribylova, M.; Vorlickova, M. A Nuclease Hypersensitive Element in the Human c-myc Promoter Adopts Several Distinct i-Tetraplex Structures. Biochem. Biophys. Res. Commun. 2000, 278, 158-166.

34. Benabou, S.; Avino, A.; Eritja, R.; Gonzalez, C.; Gargallo, R. Fundamental aspects of the nucleic acid i-motif structures. RSC Adv. 2014, 51, 26956-26980.

35. Manzini, G.; Yathindra, N.; Xodo, L.E. Evidence for intramolecularly folded i-DNA structures in biologically relevant CCCrepeat sequences. Nucleic Acids Res. 1994, 22, 4634-4640.

36. Dembska, A.; Switalska, A.; Fedoruk-Wyszomirska, A.; Juskowiak, B Development of fluorescence oligonucleotide probes based on cytosine- and guanine-rich sequences. Sci. Rep. 2020, 10, 11006.

Publisher's Note: MDPI stays neutral with regard to jurisdictional claims in published maps and institutional affiliations.

(C) 2020 by the authors. Submitted for possible open access publication under the terms and conditions of the Creative Commons Attribution (CC BY) license (http://creativecommons.org/licenses/by/4.0/). 